\title{
A Unique Case of Successful Mechanical Thrombectomy and Stenting of Cabrol Graft: A Case Report
}

\author{
Puneeth Shridhar' ${ }^{1}$, Triston Smith ${ }^{2}$, Ramzi Khalil ${ }^{3}$, David Lasorda ${ }^{3}$, Young Jae Chun ${ }^{1,4,5}$ \\ ${ }^{1}$ Department of Bioengineering, University of Pittsburgh, Pittsburgh, USA \\ ${ }^{2}$ Department of Cardiology, Wheeling Hospital, Wheeling, USA \\ ${ }^{3}$ Department of Cardiology, Allegheny General Hospital, Pittsburgh, USA \\ ${ }^{4}$ Department of Industrial Engineering, University of Pittsburgh, Pittsburgh, USA \\ ${ }^{5} \mathrm{McG}$ owan Institute for Regenerative Medicine, Pittsburgh, USA \\ Email: pus8@pitt.edu, yjchun@pitt.edu,rkhalil@wpahs.org, dlasorda@wpahs.org
}

How to cite this paper: Shridhar, P., Smith, T., Khalil, R., Lasorda, D. and Chun, Y.J. (2016) A Unique Case of Successful Mechanical Thrombectomy and Stenting of Cabrol Graft: A Case Report. World Journal of Cardiovascular Diseases, 6, 295-299. http://dx.doi.org/10.4236/wjcd.2016.69033

Received: July 22, 2016

Accepted: August 29, 2016

Published: September 2, 2016

Copyright $\odot 2016$ by authors and Scientific Research Publishing Inc. This work is licensed under the Creative Commons Attribution International License (CC BY 4.0). http://creativecommons.org/licenses/by/4.0/ (c) (i) Open Access

\begin{abstract}
Cabrol technique and its modification are used as second line or bailout procedure in reimplantation of coronary arteries after aortic root replacement. Percutaneous interventions of Cabrol graft with aspiration thrombectomy and stenting have been previously described. We report a patient who successfully underwent percutaneous intervention on the graft by rheolytic thrombectomy followed by stenting in the setting of ST elevation myocardial infarction.
\end{abstract}

\section{Keywords}

Cabrol Graft, Mechanical Thrombectomy, Manual Thrombectomy

\section{Introduction}

Interventional cardiologists are increasingly involved in percutaneous management of surgical grafts and bypasses in acute myocardial infarction. Cabrol procedure is anastomosed end-to-side to the aortic graft and end-to-end to the native coronary artery [1].

It is particularly useful when safe aorto-coronary anastomosis is hindered, such as in extreme aortic dilatation, heavily calcified aorta and re-operation. Cabrol graft occlusion is a known complication and percutaneous coronary intervention (PCI) has been performed to restore the patency of the graft.

This is a case where we report unsuccessful manual aspiration thrombectomy (AT) followed by successful mechanical (rheolytic) thrombectomy (MT) and stenting of a 
Cabrol graft in the setting of ST elevation myocardial infarction (STEMI).

\section{Case Report}

This is a 64-year-old male who presented initially with chest pain for a week and "hearing whooshing sound" in his heart. Trans-esophageal echocardiogram showed severe aortic regurgitation secondary to fibrotic and restricted right coronary leaflet with dilated aortic root and $6 \mathrm{~cm}$ aneurysmal dilation of the proximal ascending aorta. He underwent surgical evaluation and ultimately aortic valve replacement with $25 \mathrm{~mm}$ mechanical valve (St Jude Medical, MN) and aortic root replacement with $26 \mathrm{~mm} \mathrm{Da-}$ cron graft (Hemashield, Maquet, Germany) with right and left coronary reconstruction with $10 \mathrm{~mm}$ Cabrol composite graft. The patient had an uneventful postoperative recovery and he was discharged home.

\section{Procedure}

Eight months following the surgery, he presented to the emergency room of a local community hospital with chest pain radiating to his both arms of 2 hours of duration. 12 lead EKG revealed lateral ST elevations with marked ST depression. The patient was already on oral anticoagulation with an INR of 2.8. Heparin 4000 units was given intravenously. He was taken to the cardiac catheterization laboratory where he developed hypotension and multiple episodes of ventricular fibrillation requiring defibrillation, intubation and intravenous amiodarone. Bilateral femoral arterial access was obtained and 6F sheaths were placed. A $40 \mathrm{cc}$ intra-aortic balloon pump (Sensation plus, Maquet, Germany) was inserted. After a brief discussion with the cardiothoracic surgical team at the tertiary hospital, decision was made to perform primary PCI at the local hospital in view of the patient's critical condition and the inability to transfer him in a timely fashion. Aortic angiography revealed total occlusion of the left limb of Cabrol graft with TIMI 0 flow and a patent right limb of the graft with TIMI-3 flow (Figure 1).

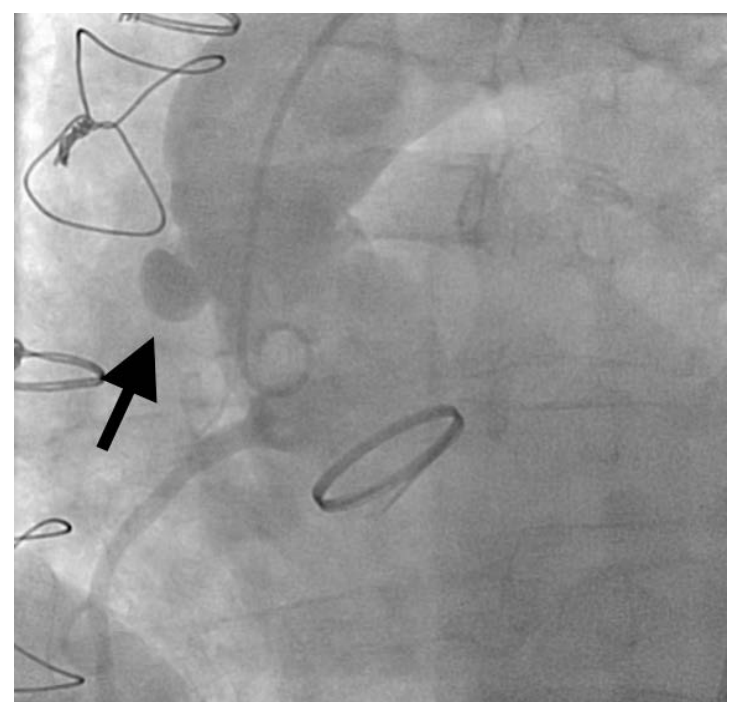

Figure 1. Aortogram in LAO view showing occlusion of the Cabrol stump (arrow). 
A 6 F JR4 guiding catheter $100 \mathrm{~cm}$ (Infiniti, Johnson and Johnson/Cordis, NJ) was used to engage the graft. A $0.014 " 50 \mathrm{~cm}$ guide wire (Pilot, Abbott Vascular, CA) was advanced and placed distally in the native left anterior descending artery. Attempt to aspirate the thrombus with the aspiration catheter (Fetch2, Medrad Inc., PA) failed to restore any flow. Decision was made to perform mechanical thrombectomy with the $4 \mathrm{~F}$ Angiojet catheter (Spiroflex, Medrad Inc., PA) two runs performed for 45 seconds each resulting in restoration of TIMI-3 flow (Figure 2). Repeat angiography showed that there was an anastomotic lesion of $75 \%$ in severity. A $4.0 \times 15 \mathrm{~mm}$ bare metal stent (Vision, Abbott Vascular, CA) was deployed at 16 atms (Figure 3). A total of 1000 mcg of intracoronary adenosine was administered. ACT by HemoTech was 280 seconds and $600 \mathrm{mg}$ Clopidogrel was loaded by the nasogastric tube at the end of procedure. The

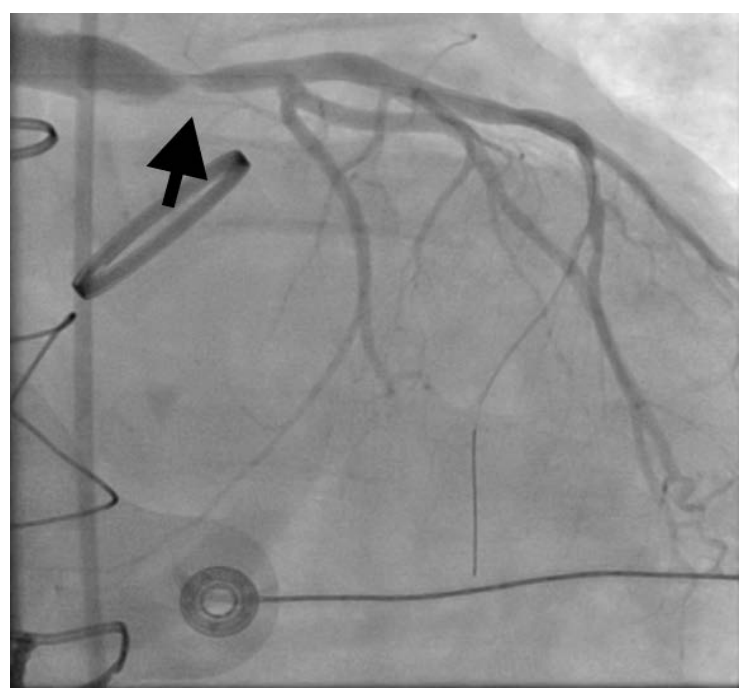

Figure 2. Pre-treatment RAO angiogram showing 75\% anastomotic lesion of left Cabrol graft/Left main trunk (arrow) with TIMI 3 flow.

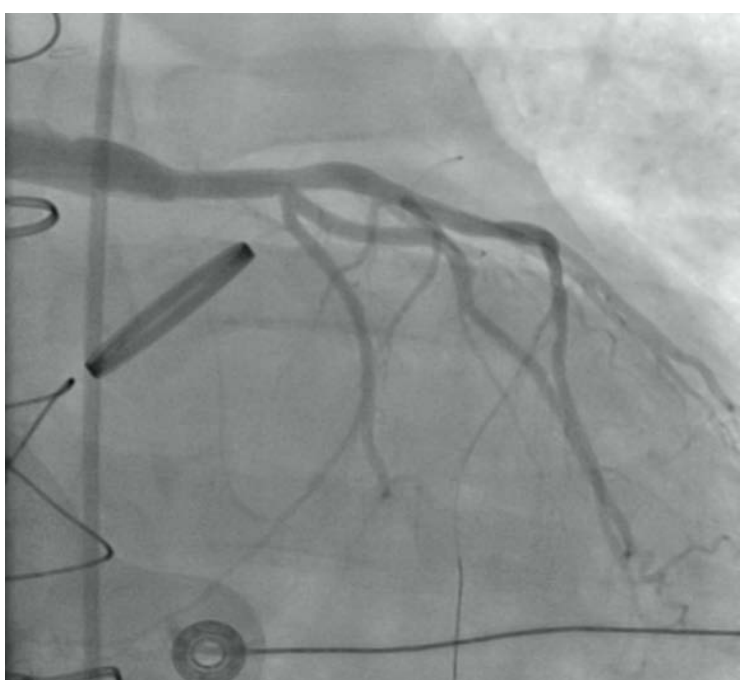

Figure 3. Post-treatment RAO angiogram view after stenting of left limb of Cabraol graft with TIMI 3 flow. 
patient was transferred to the tertiary hospital intensive care unit. He was discharged five days after the primary PCI. At one year follow up, the patient was symptom free.

\section{Discussion}

Cabrol procedure is often used when tension free anastamosis of the coronary vessels to the aortic root is not feasible. Occlusion of the graft is a known complication [2]. During the event of acute ST elevation myocardial infarction following graft closure, redo surgery is not feasible in a timely fashion. In such circumstances, primary percutaneous coronary intervention appears to be the best option. Patients undergoing PCI with stenting of Cabrol graft [2]-[4] have been described. Stent placement in the modified Cabrol graft has also been reported [5]. This a first report where MT and stenting has been successfully used for thrombus extraction from a Cabrol graft after failure of AT followed by stenting.

MT has been utilized in the setting of acute myocardial infarction in native coronaries, vein grafts, acute limb ischemia and vascular access grafts. While the AHA/ACC guidelines have favored AT in myocardial infarction as class IIa indication, the usefulness of MT in acute myocardial infarction has been controversial [6]. The original AIMI trial showed increase in major adverse cardiovascular events (MACE) and mortality with rheolytic thrombectomy compared to the conventional therapy suggesting harm. Subsequently the new JETSTENT trial (i.e., AngioJet rheoloytic thrombectomy) demonstrated that, there was improvement in early ST resolution and decrease in 6-month MACE in large vessels ( $\geq 2.5 \mathrm{~mm}$ ) and large thrombus burden (grade 3 to 5).

There have been several meta-analyses published comparing the two modalities of thrombectomy. The paper by Kumbhani et al. looking at AT vs conventional PCI and MT vs conventional PCI reported decrease in MACE and all-cause mortality with AT while there was no benefit from MT [7]. Another recent meta-analysis looked at 3 randomized controlled trials with total of 516 patients comparing head-to-head AT vs MT reported no direct benefits from MT. However, this study supported the use of MT in population with large thrombus burden [8]. Hence, the AT using an aspiration catheter would be an excellent treatment option for removing small (and may be soft) blood clots from coronary arteries, this technique is not a good method to remove severe clots in the coronary arteries and even in Cabrol graft regions. While the MT procedure is still controversial, this technique can demonstrate superior outcomes in the aspect of severe clot removal because MT typically generates pulse to deliver lytic to the locations of the thrombosed regions before mechanical suction in order to rapidly restore blood flow.

In our case, the use of AT did not restore flow most likely secondary to large thrombus burden (graft being $10 \mathrm{~mm}$ in diameter). MT was successfully used as bail out to revascularize the patient. In the absence of definitive evidence of angiographic thrombus, the AngioJet catheter may have helped to dotter open the graft. Acute closure of Cabrol grafts can be fatal, cardiologists and cardiothoracic surgeons should be aware of their severe complications. 


\section{Conclusion}

This case is unique in describing acute closure of a Cabrol graft presenting with STEMI and successful rheolytic thrombectomy and stenting.

\section{References}

[1] Cabrol, C., Pavie, A., Gandjbakhch, I., Villemot, J.P., Guiraudon, G., Laughlin, L., et al. (1981) Complete Replacement of the Ascending Aorta with Reimplantation of the Coronary Arteries: New Surgical Approach. Journal of Thoracic and Cardiovascular Surgery, 81, 309-315.

[2] Wells, T.A. and Kapoor, A. (2007) Percutaneous Coronary Intervention through a Cabrol Composite Graft. International Journal of Cardiology, 116, 44-45.

http://dx.doi.org/10.1016/j.ijcard.2006.08.101

[3] Hoskins, M.H., Kacharava, A.G., Green, T.F. and Marromatis, K. (2010) Percutaneous Intervention of Cabrol Graft-Left Main Anastomosis during Acute Myocardial Infarction. International Journal of Cardiology, 140, 27-29.

[4] Uribe González, J., Peña Duque, M.A. and Larraga, M.P. (2012) Early Stenosis in a GoreTex Graft Connected to the Left Main: An Emergency Transradial Angioplasty for a Complication in a Cabrol Procedure. Journal of Invasive Cardiology, 24, 539-542.

[5] Pappy, R.M., Hanna, E.B., Peyton, M.D. and Saucedo, J.F. (2012) Isolated Right Ventricular Infarction after Modified Cabrol Operation. Texas Heart Institute Journal, 39, 133-137.

[6] Levine, G.N., Bates, E.R., Blankenship, J.C., Bailey, S.R., Bittl, J.A., Cercek, B., et al. (2011) ACCF/AHA/SCAI Guideline for Percutaneous Coronary Intervention: A Report of the American College of Cardiology Foundation/American Heart Association Task Force on Practice Guidelines and the Society for Cardiovascular Angiography and Interventions. Journal of the American College of Cardiology, 58, 44-122. http://dx.doi.org/10.1016/j.jacc.2011.08.007

[7] Kumbhani, D.J., Bavry, A.A., Desai, M.Y., Bangalore, S. and Bhatt, D.L. (2013) Role of Aspiration and Mechanical Thrombectomy in Patients with Acute Myocardial Infarction Undergoing Primary Angioplasty: An Updated Meta-Analysis of Randomized Trials. Journal of the American College of Cardiology, 62, 1409-1418.

http://dx.doi.org/10.1016/j.jacc.2013.04.025

[8] Navarese, E.P., Tarantini, G., Musumeci, G., Napodeno, M., Rossini, R. and Kowalawski, M. (2013) Manual vs Mechanical Thrombectomy during PCI for STEMI: A Comprehensive Direct and Adjusted Indirect Meta-Analysis of Randomized Trials. American Journal of Cardiovascular Disease, 3, 146-157.

\section{Abbreviations}

$\mathrm{PCI}=$ Percutaneous Coronary Intervention, $\mathrm{AT}=$ Aspiration Thrombectomy, $\mathrm{MT}=$ Mechanical Thrombectomy, STEMI $=$ ST Elevation Myocardial Infarction, EKG = Electrocardiogram, INR = International Normalized Ratio, TIMI $=$ Thrombolysis in Myocardial Infarction, ACT = Activated Clotting Time, AHA = American Heart Association, ACC $=$ American College of Cardiology, MACE $=$ Major Adverse Cardiovascular Events 